\title{
Fórmula para la valoración monetaria del árbol urbano en Chile central
}

\author{
A formula for monetary valuation of urban trees in central Chile
}

\author{
Mauricio Ponce-Donoso a*, Óscar Vallejos-Barra ${ }^{a}$, Francisco J Escobedo $\mathbf{M}^{\mathrm{b}}$ \\ *Autor de correspondencia: a Universidad de Talca, Facultad de Ciencias Forestales, \\ Avda. Lircay s/n, Talca, Chile, tel.: (56)712200432, mponce@utalca.cl

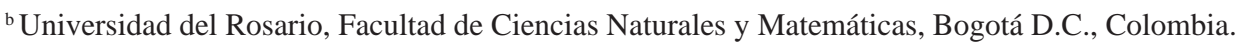

\begin{abstract}
SUMMARY
There is currently a lack of tree valuation methods to better appraise the effects, benefits and legal actions related to urban forests in South American countries; and those which have one, present difficulties of application. To address this, five parametric formulas were elaborated for urban trees appraisal in central Chile, to assess their performance against two previously evaluated formulas: the Chilean municipalities of Concepcion, La Pintana and Maipú formula (COPIMA) and the United States' Council of Tree and Landscape Appraisers (CTLA). Formulas were developed using national and international professionals and multiple relevant treesite variables that should be considered when assessing monetary value. The formulas were proposed using a focus group of national professionals to better adjust formulas using criteria related to: feasibility of application, minimum and maximum monetary values, feasibility in legal actions, lack of statistically significant differences among evaluators, tree and aesthetic variables, sites, amenities and ecosystem services and dis-services. The seven formulas were tested on 30 trees in three communes of Chile, Santiago, Talca and Concepción, by eight professionals, yielding a total sample of 1,680. Afterwards, samples were analyzed for statistical differences. Findings were used to develop a regional tree valuation formula called Valoración Monetaria del Arbolado Urbano (VaMA). The formula may be used by municipalities, professional and researches to better assess the monetary value of public and private urban trees in an efficient and cost-effective manner.
\end{abstract}

Key words: arboriculture, tree appraisal, urban forestry, tree valuation.

\section{RESUMEN}

Países en Sudamérica carecen de métodos para valorar el arbolado urbano con el fin de dar importancia a sus benéficos o bien para establecer indemnizaciones y aquellos que los tienen, presentan dificultades de aplicación. A fin de proponer una fórmula a ser usada en la valoración del arbolado urbano en Chile central, se elaboraron cinco fórmulas paramétricas, las que fueron comparadas con dos fórmulas previamente evaluadas: la chilena de los municipios de Concepción, La Pintana y Maipú (COPIMA) y la Trunk Replacement Formula del Council of Tree and Landscape Appraisers de los Estados Unidos (CTLA). Para el desarrollo de las fórmulas se consultó a profesionales, nacionales e internacionales, sobre variables del arbolado que deberían ser consideradas para valoración monetaria. Las fórmulas fueron analizadas por profesionales nacionales en un focus group, para ajustar sus parámetros en función de criterios asociados a: factibilidad de aplicación, valores monetarios mínimos y máximos de la tasación, viabilidad de concretar una indemnización, diferencias estadísticas no significativas entre evaluadores, variables dasométricas y estéticas, ubicación, amenidades, servicios y dis-servicios ecosistémicos. Las siete fórmulas se aplicaron a 30 árboles ubicados en tres comunas de Chile, Santiago, Talca y Concepción, labor que fue realizada por ocho profesionales, obteniéndose un total de 1.680 datos, que fueron analizados estadísticamente. Se seleccionó una fórmula regional para valorar el arbolado denominada valoración monetaria del arbolado urbano (VaMA). Con ella, los municipios de Chile central y profesionales que requieran determinar este valor, en sitios de uso público o privado, cuentan con una herramienta efectiva y eficaz.

Palabras clave: arboricultura, avalúo del arbolado urbano, arbolado urbano, valor del árbol.

\section{INTRODUCCIÓN}

La preocupación por la gestión del arbolado urbano y sus áreas verdes en las ciudades de Sudamérica está siendo cada vez más demandada por sus habitantes. La conceptualización nace en Norteamérica en los años '60, mientras que en Europa lo hace en los 80 (Pauleit et al. 2002, Konij- nendijk et al. 2005). Se define arbolado urbano como aquella área arbolada que se ubica tanto en zonas urbanas como periurbanas (Tyrväinen et al. 2003) constituyendo un patrimonio económico, social y ambiental para las ciudades (Tyrväinen 2001, Tyrväinen et al. 2003, Konijnendijk et al. 2004, 2005, Cullen 2005, Ponce-Donoso et al. 2009). En la actualidad variados estudios rescatan los diferentes 
servicios ecosistémicos que proveen los árboles urbanos, aspectos que le agregan valor, tales como el mejoramiento de la calidad de vida, secuestro de carbono, reducción de contaminantes y ruido, entre otros (Dobbs et al. 2011, Escobedo et al. 2011, Roy et al. 2012, Haase et al. 2014).

La determinación del valor monetario del arbolado urbano es parte de su gestión, la que es imprecisa producto del uso de diversas metodologías, de las variables que participan en ella y de la subjetividad que imprime cada evaluador (Caballer 1999, Watson 2002, Price 2003, Tyrväinen et al. 2003), a pesar que las variables que componen un método debieran presentar la menor subjetividad (Chueca 2001). Por lo general, los métodos se traducen en fórmulas, cuya aplicación se fundamenta en la necesidad de determinar su valor monetario a causa de litigios, reclamos, tasaciones, expropiaciones y determinación de daños, así como recientemente el valor de los servicios ecosistémicos que brinda el arbolado. Por ello, la determinación de su valor es un desafío permanente, que involucra procedimientos econométricos que incorpora variables ambientales, sociales, históricas, simbólicas, entre otras.

El método más usado para determinar el valor monetario del árbol urbano es la aplicación de fórmulas de valoración (Watson 2002). Es posible identificar fórmulas desarrolladas en diversos países como Argentina, Australia, Brasil, Chile, Dinamarca, España, Estados Unidos, Finlandia, Francia, Hungría, Nueva Zelanda y Reino Unido, entre otros, siendo algunas de ellas abordadas en variados estudios (Tyrväinen 2001, Watson 2002, Randrup 2005, Contato-Carol et al. 2008, Ponce-Donoso et al. 2009, Grande-Ortiz et al. 2012). Entre las más estudiadas están el método Burnley de Australia (Moore y Arthur 1992); la del Council of Tree and Landscape Appraisers de Estados Unidos (CTLA 2000); el método de valoración de amenidades de árboles y bosques de Gran Bretaña, denominado Helliwell (Watson 2002); el Standard Tree Evaluation Method, conocido como STEM de Nueva Zelanda (Flook 1996); la Norma Granada de la Asociación Española de Parques y Jardines Públicos (AEPJP 2007); como también algunas latinoamericanas, entre ellas el método Contato y COPIMA (Contato-Carol et al. 2008, Ponce-Donoso et al. 2012, 2013).

Las fórmulas para valorar monetariamente el árbol tienen por lo general una estructura de parámetros ponderados o bien, son fórmulas de capitalización (Grande-Ortiz 2012). Watson (2002) se refiere a la subjetividad de los evaluadores como la principal debilidad de las primeras, mostrando que su carácter multiplicativo aumenta estas diferencias; mientras que Contato-Carol et al. (2008) señalan que las segundas son más usadas en los municipios por su simpleza. Respecto de las primeras, estudios de Ponce-Donoso et al. (2009, 2012, 2013 y 2016) muestran que las variaciones pueden alcanzar hasta $400 \%$, aspecto que debiese ser considerado al momento de seleccionar una fórmula.

Crecientemente los municipios chilenos han desarrollado ordenanzas que buscan establecer criterios y orienta- ciones, tanto generales como particulares, respecto de las áreas verdes y arbolado de su comuna; sin embargo, no todas incorporan alguna expresión o fórmula que permita valorar el arbolado urbano, a fin de ser usada para caracterizar su patrimonio en términos pecuniarios, o bien para determinar el valor de la pérdida o daño del árbol cuando haya sido intervenido fuera del marco legal, o debido a un accidente o vandalismo. Este daño, en el sentido expuesto, es una pérdida del patrimonio comunal que representa el árbol, si se consideran los diferentes servicios ecosistémicos que aporta. Por lo anterior, la pérdida del patrimonio público o privado que representa este tipo de árbol, según sea el caso, requiere contar con técnicas que permitan determinar su importancia o valor monetario (Hegedüs et al. 2011) que bajo criterios de eficiencia y costo-eficacia, asociado a la administración pública, permita determinar el valor que busque compensar el daño causado (Grande-Ortiz et al. 2012, Ponce-Donoso et al. 2012), que en el caso chileno, se realiza a través de la actuación del Juzgado de Policía Local, instancia donde los municipios podrían recuperar esta pérdida.

La valoración monetaria del arbolado urbano es de reciente preocupación en Chile. Son los municipios quienes inicialmente llevaron a cabo acciones en este sentido, dado que el árbol se encuentra ubicado preferentemente en áreas de uso público; por lo cual, no tan solo su establecimiento, cuidado y mantención son parte del quehacer municipal, sino que también sancionar a aquellos que por sus conductas le ocasionan algún tipo de daño o pérdida. Ponce y Piedrahita (2009) identificaron variadas formas para determinar la compensación por daño al arbolado en municipios de Chile, siendo la más recurrente el uso de fórmulas, tanto de tipo paramétricas como de capitalización. Las paramétricas son consideradas más frecuente (Moore y Arthur 1992, Flook 1996, CTLA 2000, Watson 2002, Randrup 2005, AEPJP 2007, Grande-Ortiz et al. 2012), mientras que las segundas son recomendadas para el ámbito público (Contato-Carol 2008).

Luego de haber identificado fórmulas y formas de valorar, Ponce-Donoso et al. (2009) compararon el desempeño de siete fórmulas chilenas usadas en 10 municipios, tanto de capitalización como paramétricas, encontrándose importantes diferencias en el valor monetario final. Posteriormente, seleccionando las fórmulas paramétricas chilenas que tenían mejor desempeño, se les compara con otras de igual tipo usadas en otros países (Ponce-Donoso et al. 2012, 2013). En el primero se selecciona tres fórmulas nacionales y cuatro internacionales, realizando una nueva tasación, con un único tasador y 14 árboles ubicados en una única comuna, obteniéndose nuevamente diferencias significativas; esta vez se observa con claridad que COPIMA y CTLA están presentes en dos de los tres grupos conformados, con las que se obtuvieron valores medios. En el segundo se amplía el número de árboles, evaluado dos de cada especie y CTLA es usada como patrón de comparación para evaluar el desempeño de todas las fórmulas identifi- 
cadas en Chile; los resultados confirman los desempeños anteriores, identificando la fórmula usada por el municipio de Peñalolén como otra factible de profundizar en su estudio junto a CLTA y COPIMA. Si bien COPIMA muestra en los estudios que tiene un desempeño similar a CTLA, debía ser comparada con un mayor número de evaluadores y se observó que adolece de variables ambientales.

Ponce-Donoso y Vallejos-Barra (2016) utilizaron nuevas fórmulas, pero esta vez en 30 árboles ubicados en 3 comunas de Chile, participan 4 tasadores, donde incluyeron las tres fórmulas destacadas anteriormente (COPIMA, CTLA y Municipalidad de Peñalolén), las que se aplicaron junto a otras nueve, todas ellas de otros países (Métodos Danés, Francés, Suizo, Italiano, Tedesco, Norma Granada, Burnley, Helliwell y STEM). Los resultados muestran que no hay diferencias significativas entre tasadores, mientras que sí lo hay para las fórmulas. Fue posible identificar que las fórmulas chilenas COPIMA y Municipalidad de Peñalolén y las internacionales CTLA, Métodos Italiano y Suizo presentan resultados estadísticamente similares, con valoraciones ubicadas en montos medios.

Los resultados de estas aplicaciones muestran amplias dispersiones y diferencias significativas, desafiando la construcción de un instrumento local que permita su aplicación en base a criterios de eficiencia y eficacia, tanto para profesionales del sector, como para las actuaciones en el contexto público, especialmente municipal y judicial; mostrando la dificultad en el uso de una única fórmula, ya sea a nivel regional, de una parte del territorio o de carácter nacional, que se pueda concretar ampliando el uso de una fórmula nacional o bien adscribiendo una internacional (Watson 2002, Randrup 2005, Contato-Carol et al. 2008, Grande-Ortiz et al. 2012).

El objetivo de este estudio es proponer una fórmula para valorar monetariamente el árbol urbano que incluya variables ambientales, a partir de la evaluación de un grupo de fórmulas, cinco construidas usando métodos participativos (consulta a expertos y focus group) y dos ya existentes y conocidas en la literatura, una nacional y otra internacional. Se plantea la hipótesis que es posible seleccionar una de las fórmulas construidas, que sea incorporada a las Ordenanzas Municipales en comunas de las regiones de Chile central; así como aplicada por profesionales e investigadores que realizan este tipo de valoraciones. Además, se incluye la hipótesis que los evaluadores del arbolado urbano presentan resultados similares.

\section{MÉTODOS}

Para la evaluación de las fórmulas se consideró el arbolado urbano de tres comunas capitales regionales ubicadas en la zona central de Chile. La primera, Santiago, se

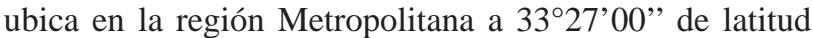
sur y $70^{\circ} 40^{\prime} 00^{\prime \prime}$ de longitud oeste, a 579 m s.n.m., cuenta con una población de 220,8 mil habitantes, concentrándose en una superficie de $22,4 \mathrm{~km}^{2}$. La comuna de Talca se ubica en la región del Maule y cuenta con una población de 201,8 mil habitantes que se distribuyen en una superficie de $232 \mathrm{~km}^{2}$, está situada a $35^{\circ} 25^{\prime} 59^{\prime \prime}$ de latitud sur y $71^{\circ} 40^{\prime} 00^{\prime \prime}$ de longitud oeste a $102 \mathrm{~m}$ s.n.m. Concepción es una comuna de la región del Biobío ubicada a 3650’00" de latitud sur y $73^{\circ} 03^{\prime} 00^{\prime \prime}$ de longitud oeste, cuenta con una superficie de $221,6 \mathrm{~km}^{2}$, concentrando una población de 216,1 mil habitantes y se localiza en las cercanías del Océano Pacífico a 12 m s.n.m., la separa de Santiago 449,7 $\mathrm{km}$ hacia el norte (BCN 2013).

Atendidas las sugerencias de Ponce-Donoso et al. (2012) en torno a aumentar la base de evaluaciones y evaluadores, en cada una de las comunas se seleccionaron 10 árboles (cuadro 1) considerando su ubicación y condición sanitaria, los que fueron tasados por ocho profesionales, de los cuales cuatro presentan experiencia de más de cinco años en este tipo de valoraciones y los restantes menores a un año. De ellos, siete son ingenieros forestales y uno ingeniero agrónomo con experiencia en valoración, conformándose dos grupos con cuatro evaluadores cada uno, sénior y júnior, dependiendo de los años de experiencia en valoración. El periodo de aplicación de las fórmulas propuestas fue entre los meses de diciembre de 2014 y enero de 2015.

Dos fórmulas evaluadas en estudios anteriores (PonceDonoso et al. 2009, 2012, 2013), la internacional del Council of Tree and Landscape Appraisers de Estados Unidos (CTLA) y la nacional aplicada en los municipios de Concepción, La Pintana y Maipú de Chile (COPIMA), sirvieron de comparación a las cinco fórmulas paramétricas propuestas, las que consideraron los siguientes criterios: a) que incluyan variables de funciones ecosistémicas; b) que las variables permitan una aplicación práctica de la fórmula, preferentemente a nivel de municipios; c) que el valor monetario final permita una real capacidad de resarcimiento por la pérdida del árbol, haciéndola viable en los Juzgados de Policía Local, tanto en su valor mínimo como máximo; d) que su valor monetario no presente diferencias significativas con la fórmula CTLA o COPIMA; y finalmente, e) se reduzcan las diferencias significativas entre tasadores y grupos de tasadores que fueron encontradas en Ponce-Donoso et al. (2012, 2013) y Ponce-Donoso y Vallejos-Barra (2016). Las fórmulas evaluadas y sus características se describen a continuación (se expresan en dólares de Estados Unidos para efectos prácticos). En el Apéndice A se presenta un cuadro comparativo de las características de las fórmulas.

El método CTLA considera el área de la sección transversal del tronco a 1,4 m sobre el nivel del suelo, multiplicado por un valor basado en el costo de la disponibilidad de la especie en viveros regionales. El valor es multiplicado por factores tales como condición, ubicación y calidad, usando una ponderación entre 0,0 a 1,0 para cada factor:

Valor $\left(\right.$ US\$) = (área del tronco $\left(\mathrm{cm}^{2}\right) *$ precio básico $\left.\mathrm{cm}^{-2}\right)$

* especie * condición * localización 
La fórmula de COPIMA es la siguiente:

$$
\text { Valor }(\mathrm{US} \$)=(\mathrm{A} * \mathrm{~B} * \mathrm{C} * \mathrm{D}) / 10
$$

Donde: $\mathrm{A}=$ precio de la especie en el mercado minorista. Las especies deben tener entre 12 a $14 \mathrm{~cm}$ de perímetro a la altura del cuello. La altura considerada para las especies de hoja persistente es de 3,5 a 4,0 m y para coníferas y palmeras de 2,0 a 2,5 m. B = Valor estético y de sanidad del árbol, cuyo rango va de 1 a 10 y considera, dependiendo de la belleza del árbol, la relación con otros árboles, protección, sanidad, vigor y valor dendrológico. C = Índice de situación.

Cuadro 1. Identificación de los árboles en las comunas de Santiago, Talca y Concepción, Chile. Identification of trees in communes of Santiago, Talca and Concepción, Chile.

\begin{tabular}{|c|c|c|c|c|c|c|}
\hline \multirow{2}{*}{$\mathrm{N}^{\circ *}$} & \multirow{2}{*}{ Especie } & \multirow{2}{*}{ Ubicación** } & \multirow{2}{*}{$\begin{array}{c}\text { Edad } \\
\text { (Años) }\end{array}$} & \multirow{2}{*}{$\begin{array}{c}\text { Condición } \\
\text { sanitaria }\end{array}$} & \multicolumn{2}{|c|}{ Variables dendrométricas } \\
\hline & & & & & $\operatorname{DAP}(1,3 \mathrm{~m})$ & Altura (m) \\
\hline 1 & Melia azedarach L. & $\mathrm{CP}$ & 30 & Buena & 36,7 & 12,2 \\
\hline 2 & Gleditsia triacanthos L. & CS & 30 & Buena & 17,8 & 8,2 \\
\hline 3 & Acacia dealbata Link & СР & 30 & Buena & 29,4 & 8,4 \\
\hline 4 & Acer pseudoplatanus L. & СР & 40 & Mediana & 24,1 & 10,7 \\
\hline 5 & Acer negundo L. & $\mathrm{CP}$ & 20 & Buena & 12,2 & 6,9 \\
\hline 6 & Platanus orientalis L. & CS & 30 & Mediana & 17,6 & 11,5 \\
\hline 7 & Fraxinus excelsior L. & CS & 50 & Buena & 26,9 & 9,8 \\
\hline 8 & Catalpa bignonioides Walt. & PS & 75 & Buena & 52,1 & 13,9 \\
\hline 9 & Liquidambar styraciflua $\mathrm{L}$. & PJ & 9 & Buena & 6,5 & 5,1 \\
\hline 10 & Koelreuteria paniculata Laxm. & AP & 35 & Buena & 19,2 & 5,2 \\
\hline 11 & Acer negundo L. & СР & 24 & Mediana & 39,3 & 9,4 \\
\hline 12 & Ailanthus altissima (Mill.) Swingle & АР & 25 & Buena & 51,9 & 18,8 \\
\hline 13 & Ginkgo biloba L. & PM & 111 & Buena & 90,7 & 22,2 \\
\hline 14 & Liquidambar styraciflua $\mathrm{L}$. & PP & 7 & Buena & 6,9 & 3,8 \\
\hline 15 & Catalpa bignonioides Walt. & $\mathrm{CP}$ & 25 & Baja & 21,8 & 6,3 \\
\hline 16 & Quercus robur L. & AP & 60 & Mediana & 77,7 & 15,1 \\
\hline 17 & Acacia melanoxylon R. Br. & PS & 28 & Buena & 30,0 & 11,3 \\
\hline 18 & Platanus orientalis L. & СР & 100 & Buena & 77,5 & 25,9 \\
\hline 19 & Betula pendula Roth & CS & 12 & Mediana & 9,1 & 5,5 \\
\hline 20 & Acacia dealbata Link & CS & 32 & Buena & 40,3 & 7,0 \\
\hline 21 & Quercus robur L. & PM & 60 & Buena & 29,8 & 10,4 \\
\hline 22 & Liquidambar styraciflua $\mathrm{L}$. & СР & 45 & Buena & 47,9 & 14,2 \\
\hline 23 & Acer negundo L. & CS & 45 & Mediana & 20,5 & 8,7 \\
\hline 24 & Acer pseudoplatanus L. & PQ & 72 & Buena & 83,4 & 15,7 \\
\hline 25 & Fraxinus americana L. & PQ & 40 & Mediana & 66,9 & 12,4 \\
\hline 26 & Platanus orientalis L. & СР & 80 & Buena & 65,3 & 18,0 \\
\hline 27 & Acacia melanoxylon R. Br. & СР & 35 & Buena & 36,4 & 10,4 \\
\hline 28 & Betula pendula Roth & PS & 20 & Baja & 38,5 & 6,4 \\
\hline 29 & Ailanthus altissima (Mill.) Swingle & PS & 35 & Buena & 66,2 & 18,1 \\
\hline 30 & Catalpa bignonioides Walt. & $P Q$ & 25 & Baja & 29,3 & 8,7 \\
\hline
\end{tabular}

* Árboles 1 al 10 se ubican en la comuna de Santiago, 11 al 20 en Talca, 21 al 30 en Concepción.

** AP: Avenida principal; CP: Calle principal; CS: Calle secundaria; PM: Plaza de Armas; PQ: Parque; PS: Plaza secundaria; PJ: Pasaje; PP: Paseo peatonal. 
Aspecto relacionado con la evaluación ambiental y la urbanización. Puede alcanzar valores de 6, 8 o 10. D = Índice de dimensión, que corresponde al perímetro del árbol medido a 1,3 m sobre el nivel del suelo, con valores que van de 1 a 20 .

Las fórmulas propuestas fueron desarrolladas a partir de dos instancias. La primera corresponde a la aplicación de un cuestionario que fuera respondido por 20 profesionales, nacionales e internacionales, ligados al arbolado urbano, que en promedio presentan más de 10 años de experiencia, a quienes se les preguntó acerca de qué variables debiesen estar presentes en una fórmula para valorar monetariamente el arbolado urbano y su impacto en la determinación del valor (Proyecto Fondecyt 1130264), lo que permitió identificar variables y su potencial influencia en el valor monetario. A partir de los resultados se logró construir cuatro fórmulas paramétricas. La segunda etapa, corresponde a la revisión de las cuatro fórmulas por parte de tres profesionales nacionales, que cuentan con más de 10 años de experiencia, que no fueron consultados en la primera instancia. Las observaciones hechas a las fórmulas fueron discutidas en un focus group con estos profesionales, junto a los investigadores del proyecto señalado, instancia que permitió ajustar las fórmulas y el surgimiento de una quinta propuesta.

La fórmula 1, propuesta en esta evaluación es:

$$
\text { Valor }(\mathrm{US} \$)=\mathrm{UTM} * \mathrm{~T} * \mathrm{U} * \mathrm{~S} * \mathrm{~A} * \mathrm{E} * \mathrm{FE} * \mathrm{DS}
$$

Donde: UTM $^{1}$ es la Unidad Tributaria Mensual del mes en que se realiza la valoración. T = Tamaño, que se expresa como una relación entre el DAP y el volumen de copa, expresado en metros cúbicos, que es además corregido de acuerdo a la forma. $U$ = ubicación, que considera a la especie emplazada en un sitio correcto, adecuado suelo, alcorque y espacio. $\mathrm{S}$ = Condición sanitaria del árbol, donde se busca identificar la vigorosidad que presenta el espécimen, tanto en la copa, fuste, ramas y raíces que están a la vista. A = Amenidades, donde se evalúa las características plásticas del árbol tanto individualmente o como grupo, incorporando aspecto de floración, fragancia y otras; puede tomar el valor de 1,5; 2,4 ó 3,0. E = Características Ambientales, relacionadas con el aporte a la biodiversidad, reducción del ruido, reducción del material particulado, reducción de gases de efecto invernadero, mejora de la humedad ambiental, reducción de los rayos ultravioleta y disminución de la erosión; el valor fluctúa entre 1,0 y 3,0 . FE $=$ Factores Especiales, que corresponde a la ponderación para ampliar el valor del árbol al identificar variables especiales a destacar, tales como: ubicación en un arboretum, árbol de componente histórico (debidamente documentado), re-

UTM: corresponde a una expresión que representa un valor monetario, el que se reajusta mensualmente en función de la variación de precios al consumidor (IPC). Los resultados se obtuvieron con la UTM del mes de marzo de 2014, equivalente a CL\$ 41.263 . licto, fuente de calidad genética u otro. Por cada uno de estos se podrá ponderar proporcionalmente en un $5 \%$, con un máximo de $30 \%$. DS = Dis-servicios, corresponde a la ponderación para reducir el valor del árbol al identificar variables que generan problemas, tales como alergias, caída de hojas, caída de frutos, rotura de infraestructura u otros. Se podría descontar un máximo del $10 \%$.

La fórmula 2, propuesta en esta evaluación, es:

$$
\text { Valor }(\mathrm{US} \$)=\mathrm{VB} * \mathrm{~T} * \mathrm{E} * \mathrm{C} * \mathrm{~L} * \mathrm{FA}
$$

Donde: VB = Valor Básico, equivalente a un décimo del precio al detalle de la planta en viveros locales de las siguientes medidas o su proporción, diámetro a la altura del cuello de $4 \mathrm{~cm}$ y una altura de $2,5 \mathrm{~m}$. T = Tamaño, expresado como la relación entre el DAP (medido en centímetros a 1,3 metros desde el nivel de suelo) y el volumen de copa (VC), medida como el volumen en metros cúbicos entre la altura y el área de proyección de la copa, que es corregida de acuerdo su forma; puede tomar valores entre $0,05 \mathrm{y}$ 1,00. $\mathrm{E}=$ Especie, que se relaciona con la especie que se evalúa, tanto por su origen, valorándose más las nativas que las exóticas, su menor presencia, como otras características comparativas respecto de otras especies presentes en la ciudad, su ponderador va de $1 \mathrm{a} 10 . \mathrm{C}=$ Composición, que corresponde a su ubicación relativa que el árbol tiene en el respecto de otros, los valores van de 2 a 10 . L = Localización, corresponde al lugar que el árbol ocupa respecto de su desarrollo y potencialidades que muestra, toma valores que van de 1 a 10. FA = Factores Ambientales, identificando el aporte a la biodiversidad, reducción del ruido, reducción del material particulado, reducción de gases con efecto invernadero, mejora de la humedad ambiental, reducción de los rayos ultravioletas y disminución de la erosión, puede tomar valores que van de 1,5 a 10 .

La fórmula 3, propuesta en esta evaluación, es:

$$
\text { Valor }(\mathrm{US} \$)=\mathrm{VB} * \mathrm{CU} * \mathrm{E} * \mathrm{~N} * \mathrm{~A} * \mathrm{FA}
$$

Donde: VB = Valor Base, queda determinado por el producto de un décimo del precio de la especie en viveros locales por centímetro cuadrado medido a la altura del cuello y el área del DAP del árbol a valorar medido a 1,4 m del suelo en centímetros cuadrados. CU = Condición y Ubicación, que corresponde a las características del árbol relacionadas con su estado sanitario y dónde se encuentra ubicado, puede tomar valores entre 0,25 a $1,0 . \mathrm{E}=$ Estado, el cual se relaciona con variables dendrométricas y características morfológicas del árbol, relacionado el DAP y la altura del copa, puede tomar valores entre 0,2 a $1,0 . \mathrm{N}=$ Notabilidad, que reconoce alguna característica destacable de la especie a evaluar, amplificando el valor monetario del árbol relacionando la especie con variables de: ubicación destacada, por ejemplo su ubicación en un arboretum, representativi- 
dad, rareza botánica, calidad genética, relicto, componente histórica u otro; monto máximo que puede aumentar al valor final obtenido es de hasta un $20 \%$. A= Amenidades, que se la relaciona con características plásticas de alineación, silueta, color, como también fragancia, floración y otra que presenta la especie; puede tomar valores de 1, 0,85 y 0,5. $\mathrm{FA}=$ Factores Ambientales. Correspondiente a variables que se le relacionan como el aporte que el espécimen hace, tal como a la biodiversidad, reducción del ruido, reducción del material particulado, reducción de gases con efecto invernadero, mejora de la humedad ambiental, reducción de los rayos ultravioletas y disminución de la erosión; puede tomar valores entre 0,3 y 1,0 .

La fórmula 4, propuesta en esta evaluación, es:

$$
\begin{gathered}
\text { Valor (US\$) }=0,000016 * \mathrm{DAP}^{2} * \mathrm{~h} * \$ / \\
\left(\mathrm{DAP}^{2} * \mathrm{~h}\right) * \mathrm{D} * \mathrm{C} * \mathrm{~L}
\end{gathered}
$$

Donde: $0,000016=\pi /(40.000 * 2,5) * 0,5=$ volumen $*$ fracción de carbono. DAP = Diámetro del tronco a 1,3 m sobre el nivel del suelo en $\mathrm{cm}$. $\mathrm{h}$ = Altura del árbol, en m. \$ = Precio de la planta en viveros mayoristas. $\mathrm{D}$ = Densidad, correspondiente a la densidad de la madera de la especie o familia. Las especies que presentan alta densidad son aquellas cuyo valor supera los $0,66 \mathrm{~g} \mathrm{~cm}^{-3}$, las de densidad media son aquellas cuya densidad es mayor a $0,5 \mathrm{~g} \mathrm{~cm}^{-3}$ y menores a la densidad alta, y las especies consideradas poco densas, son aquellas que tienen un valor menor a 0,5 $\mathrm{g} \mathrm{cm}^{-3}$. El índice será de 600 para especies de alta densidad, 400 para densidad media y de 200 para poco densas. C = Condición, variable que se relaciona con características de sanidad, estructura y crecimiento del árbol, evaluando la sanidad en general, estructura de copa, ramas sanas, mantención, color del follaje y crecimiento; puede tomar valores entre 0,2 a 1,0. $\mathrm{L}=$ Localización, donde se pondera al árbol en función de su ubicación en la ciudad, desde áreas residenciales a bosque periurbanos, su valor va de 0,2 a 1,0.

La fórmula 5, propuesta en esta evaluación, es:

Valor $(\mathrm{US} \$)=\mathrm{UTM} * \mathrm{~T} * \mathrm{E} * \mathrm{U} * \mathrm{C} * \mathrm{FA} * \mathrm{CP} * \mathrm{FE}$

Donde: UTM = Unidad Tributaria Mensual del mes cuando en que se realiza la valoración. $\mathrm{T}$ = Tamaño, expresado como la relación del DAP y el volumen de copa, esta último corregido de acuerdo a su forma; sus valores van de 0,05 a 1,0. E = Especie, variable que relaciona su origen, adaptación al medio urbano, necesidad de manejo especial y representación en la ciudad; puede tomar un valor de 1,0 a 3,0. U = Ubicación, que pondera si el árbol se encuentra ubicado en un lugar adecuado para su desarrollo en consideración a sus potencialidades; su valor oscila entre 0,3 a 3,0. C = Condición, corresponde a la condición sanitaria del árbol, rescatando la vigorosidad de la copa, fuste, ramas y raíces; su valor puede ir de 0,6 a 3,0. FA = Factores ambientales, que busca reconocer el potencial aporte del árbol en términos de biodiversidad, amortiguación del ruido, reducción del material particulado, disminución de los gases de efecto invernadero, mejora de la humedad ambiental, reducción de los rayos ultravioleta y disminución de la erosión; puede tener un valor de 1,0 a 3,0. CP = Composición en el paisaje, identificando las características del árbol respecto de otros árboles presentes en el entorno de influencia; su valor puede ir de 1,0 a 3,0. FE $=$ Factores especiales, corresponde a un ponderador por una o más variables que se relacionan por algún aspecto destacado no considerado en las anteriores, que puede ir desde la presencia del árbol en un arboretum, aporte histórico, relicto, en peligro, fuente de calidad genética u otro; su ponderación es libre, pudiendo ir hasta un $30 \%$.

Para determinar si existían diferencias significativas entre las diferentes fuentes de variación, esto es fórmulas, grupo de tasadores y tasadores individuales, se utilizó análisis de varianza paramétrico y no paramétrico, a fin de discriminar de mejor manera la selección de la fórmula y el comportamiento que presentan los tasadores en esta elección. El análisis paramétrico considera el promedio de la valoración monetaria, mientras que el no paramétrico utiliza el promedio del ranking del ordenamiento ascendente de las valoraciones, para lo cual el primer término del ranking, es decir el $N^{\circ} 1$ corresponde a la menor valoración (US\$ 0,5) y el último término del ranking, esto es el $\mathrm{N}^{\circ} 1.680$, es para el mayor valor (US\$ 26.850,17); la conversión de valor a un ranking tiene por objeto disminuir la influencia de los valores extremos (máximos o mínimos), que éstos presentan en el promedio utilizado en el análisis de varianza paramétrico.

En ambos casos, hubo diferencias significativas entre las fuentes de variación, por lo que se aplicó la prueba de comparación múltiple de Tukey HSD. La excepción fue la comparación de los dos grupos de tasadores, que no presentaron diferencias estadísticas. Para el análisis de los datos se usaron los programas Microsoft Excel v.2003 y Statgraphics Centurión v.XVII, de Windows.

Para determinar dichas diferencias, se plantearon las siguientes hipótesis, tanto para las fórmulas evaluadas, los grupos de evaluadores y los evaluadores individuales:

H0: $\omega \mathrm{i}=\omega \mathrm{j} / \mathrm{i} \neq \mathrm{j}$; es decir, no existen diferencias significativas entre las medianas de las fórmulas, grupo de evaluadores y evaluadores individuales.

$\mathrm{H} 1: \omega \mathrm{i} \neq \omega \mathrm{j} / \mathrm{i} \neq \mathrm{j}$; es decir, existen diferencias significativas entre las medianas de las fórmulas, grupo de evaluadores y evaluadores individuales.

\section{RESULTADOS}

El promedio de las valoraciones por individuo (cuadro 2) mostró que en general los árboles con características más destacadas tuvieron valores más altos, como es el caso del G. biloba (árbol № 13), y aquellos que las presentaban más disminuidas fueron valorados con montos más pequeños, tales como la $B$. pendula $\left(\mathrm{N}^{\circ} 19\right)$ y 
C. bignonioides ( $\left.\mathrm{N}^{\circ} 30\right)$. Donde no se observa claramente lo anterior es con la B. pendula ( $\left.\mathrm{N}^{\circ} 28\right)$ que siendo un árbol con problemas sanitarios, su valor final no fue comparativamente más bajo que otros de similares características, debido quizás a su ubicación; situación inversa ocurrió con la K. paniculata $\left(\mathrm{N}^{\circ} 10\right)$ que aun siendo una especie poco representada en la ciudad y ubicada en un lugar destacado, logró un valor más bien moderado.
Los estadígrafos (cuadro 3) permitieron observar los valores y ranking en forma global. Los valores promedios tuvieron cierta similitud, aunque las fórmulas 3, 4 y 5 se presentaron con valores más altos comparativamente, aunque la transformación de valor a un ranking logró aminorar el impacto de los valores extremos de estas fórmulas. La fórmula 5 fue la única que mantuvo un promedio elevado. Se observa que los coeficientes de variación disminuyen en el ranking,

Cuadro 2. Valor promedio (US\$) en tres comunas de Chile central.

Appraisal output (average, US\$) in three communes of central Chile.

\begin{tabular}{|c|c|c|c|c|c|c|c|}
\hline Árbol & CTLA* & COPIMA** & F 1 & F 2 & F 3 & F 4 & F 5 \\
\hline 1 & 424,4 & 655,4 & 678,4 & 499,0 & 371,6 & 704,1 & 997,6 \\
\hline 2 & 493,8 & 896,0 & 699,9 & 792,0 & 196,0 & 627,1 & $1.129,4$ \\
\hline 3 & 532,3 & 894,5 & 806,7 & 805,8 & 806,5 & 729,8 & $1.071,3$ \\
\hline 4 & 543,3 & 992,5 & 927,3 & 881,3 & 252,0 & 846,3 & $1.383,9$ \\
\hline 5 & 95,5 & 480,1 & 425,1 & 210,0 & 19,6 & 186,9 & 639,1 \\
\hline 6 & 440,5 & 812,3 & 752,3 & 692,5 & 204,3 & 585,3 & $1.137,2$ \\
\hline 7 & 435,4 & 681,6 & 753,8 & 544,8 & 282,9 & 482,8 & $1.130,0$ \\
\hline 8 & $1.055,0$ & $1.156,7$ & $1.038,8$ & 949,1 & $1.221,0$ & 879,1 & $1.214,7$ \\
\hline 9 & 125,6 & 421,0 & 213,4 & 206,2 & 24,8 & 83,0 & 428,1 \\
\hline 10 & 685,5 & $1.276,6$ & 658,2 & 796,8 & 352,8 & 353,1 & 998,3 \\
\hline 11 & 495,5 & 817,1 & 630,1 & 435,1 & 398,3 & 409,4 & 997,6 \\
\hline 12 & $1.052,8$ & $1.117,6$ & $1.387,8$ & $1.133,9$ & $1.216,4$ & $1.388,9$ & $1.568,5$ \\
\hline 13 & $1.628,6$ & $1.569,9$ & $1.519,2$ & $1.592,3$ & $1.670,8$ & $1.669,0$ & $1.640,8$ \\
\hline 14 & 131,0 & 478,1 & 334,9 & 333,7 & 50,5 & 118,3 & 556,1 \\
\hline 15 & 168,9 & 725,6 & 751,9 & 572,5 & 663,3 & 416,3 & 902,2 \\
\hline 16 & $1.303,3$ & $1.353,7$ & $1.125,8$ & $1.199,4$ & $1.610,4$ & $1.634,9$ & $1.392,8$ \\
\hline 17 & 646,1 & 869,6 & $1.044,7$ & 703,3 & 714,0 & 993,5 & $1.356,7$ \\
\hline 18 & $1.381,8$ & $1.449,2$ & $1.111,3$ & $1.056,4$ & $1.489,1$ & $1.600,4$ & $1.326,0$ \\
\hline 19 & 116,5 & 358,7 & 230,4 & 152,3 & 30,3 & 101,4 & 386,9 \\
\hline 20 & 715,4 & $1.013,9$ & 798,5 & 502,1 & 959,0 & 979,4 & $1.055,9$ \\
\hline 21 & 787,9 & $1.121,1$ & 865,0 & 1057,1 & 825,5 & $1.214,8$ & $1.158,6$ \\
\hline 22 & $1.324,8$ & $1.454,5$ & $1.050,1$ & 1163,3 & $1.068,6$ & $1.479,9$ & $1.391,4$ \\
\hline 23 & 271,1 & 660,3 & 706,3 & 407,3 & 119,4 & 350,8 & 953,4 \\
\hline 24 & $1.313,8$ & $1.243,1$ & $1.401,3$ & $1.338,0$ & $1.590,6$ & $1.551,1$ & $1.545,6$ \\
\hline 25 & $1.182,3$ & $1.134,4$ & $1.396,4$ & $1.226,8$ & $1.448,4$ & $1.198,8$ & $1.577,4$ \\
\hline 26 & $1.269,1$ & 1411,3 & $1.173,4$ & $1.113,6$ & $1.417,5$ & $1.560,9$ & $1.424,8$ \\
\hline 27 & 622,3 & 900,8 & 643,5 & 451,4 & 614,4 & 888,9 & 913,2 \\
\hline 28 & 429,9 & 917,1 & 427,2 & 254,1 & 575,3 & 673,5 & 504,9 \\
\hline 29 & 987,0 & $1.077,0$ & $1.317,8$ & $1.121,1$ & $1.283,3$ & $1.414,4$ & $1.538,8$ \\
\hline 30 & 221,5 & 706,3 & 221,7 & 85,8 & 258,3 & 205,3 & 229,3 \\
\hline
\end{tabular}

*CTLA: fórmula del Council of Tree and Landscape Appraisers de los Estados Unidos.

**COPIMA: fórmula usada por los municipios de Concepción, La Pintana y Maipú. 
ya que el efecto de los valores extremos tanto en la desviación estándar como en el promedio se ven aminoradas.

Evaluación de fórmulas. Se encontraron diferencias significativas en las fórmulas evaluadas, tanto en valor (estadígrafo de 11,90, $P<0,01$ ) como en el ranking (estadígrafo de 21,30, $P<0,01)$. Las fórmulas se agruparon en dos grupos homogéneos al considerar el valor (grupo A y B, cuadro 4), mientras que para el ranking fueron cuatro grupos homogéneos (grupo C, D, E y F, cuadro 4). Las fórmulas propuestas tuvieron resultados disímiles en términos monetarios, al compararlas con COPIMA y CTLA; así estas últimas en valor no presentan diferencias significativas con las fórmulas 1 y 2 (cuadro 4 y figura 1A); mientras que en el ranking CLTA, cuyos valores se ubicaron en la parte baja, conformó grupos con las fórmulas 2 y 3 (cuadro 4 y figura 1B) y COPIMA fue parte de otro grupo, con la fórmulas 1 y 4 .
La influencia de los valores máximos de la fórmula 3, 4 y 5 (figura 1A), origina que se agrupen en el grupo B (cuadro 4), lo que conlleva un promedio mayor. Esta influencia decrece en el ranking (figura 1B) y logra que la fórmula 3 presente el menor promedio junto a la fórmula 2 y CTLA (cuadro 4). La fórmula 3 presenta la menor mediana y con ello el ranking promedio resulta ser menor, ya que disminuye la influencia de los valores extremos.

Diferencias entre grupos de evaluadores. Ambos análisis de varianza, del valor y del ranking, no mostraron diferencias significativas en los grupos de evaluadores (los estadígrafos fueron de 0,10 para el valor y 0,50 para el ranking, ambos con $P>0,05)$. Para el valor se conformó el grupo $\mathrm{G}$ y para el ranking el grupo $\mathrm{H}$ (cuadro 5). La dispersión del grupo de evaluadores entre el valor (figura 1A) y el ranking (figura 1B) es prácticamente idéntico.

Cuadro 3. Estadígrafos del valor y ranking por fórmula.

Statistics of value and ranking by formula.

\begin{tabular}{llrrrrrrr}
\hline Variable & Estadígrafo & CTLA & COPIMA & F 1 & F 2 & F 3 & F 4 & F 5 \\
\hline \multirow{5}{*}{ Valor (US\$) } & Mínimo & 12,3 & 79,4 & 24,5 & 0,5 & 1,6 & 12,6 & 3,6 \\
& Promedio & $1.016,5$ & $1.048,1$ & 898,1 & 836,2 & $1.852,5$ & $2.094,1$ & $2.127,6$ \\
& Mediana & 291,9 & 657,1 & 511,7 & 418,2 & 276,9 & 457,9 & $1.210,8$ \\
& Máximo & $17.411,8$ & $5.714,3$ & $6.798,2$ & $8.465,6$ & $26.682,5$ & $26.850,2$ & $16.995,5$ \\
& CV* & 217 & 101 & 112 & 149 & 219 & 198 & 117 \\
\hline \multirow{3}{*}{ Ranking } & Mínimo & 42,0 & 239,0 & 82,0 & 1,5 & 3,0 & 43,0 & 11,5 \\
& Promedio & 696,0 & 954,9 & 836,4 & 742,6 & 724,5 & 844,2 & $1.085,0$ \\
& Mediana & 587,5 & 949,5 & 821,0 & 731,8 & 569,5 & 779,5 & $1.198,3$ \\
& Máximo & $1.670,0$ & $1.591,0$ & $1.621,0$ & $1.637,0$ & $1.679,0$ & $1.680,0$ & $1.669,0$ \\
\hline
\end{tabular}

*Coeficiente de variación.

Cuadro 4. Prueba de Tukey HSD del valor y ranking por fórmula.

Tukey HSD test of value and ranking by formula.

\begin{tabular}{|c|c|c|c|c|}
\hline Fórmula & $\begin{array}{c}\text { Valor } \\
\text { promedio (US\$) }\end{array}$ & $\begin{array}{c}\text { Grupos } \\
\text { homogéneos del valor }\end{array}$ & $\begin{array}{l}\text { Ranking } \\
\text { Promedio }\end{array}$ & $\begin{array}{c}\text { Grupos } \\
\text { homogéneos del ranking }\end{array}$ \\
\hline CTLA & $1.016,5$ & A & 696,0 & C \\
\hline COPIMA & $1.048,1$ & A & 954,9 & $\mathrm{E}$ \\
\hline 1 & 898,1 & A & 836,4 & D E \\
\hline 2 & 836,2 & A & 742,6 & C D \\
\hline 3 & $1.852,5$ & B & 724,5 & C D \\
\hline 4 & $2.094,1$ & B & 844,2 & D E \\
\hline 5 & $2.127,6$ & B & $1.085,0$ & F \\
\hline
\end{tabular}



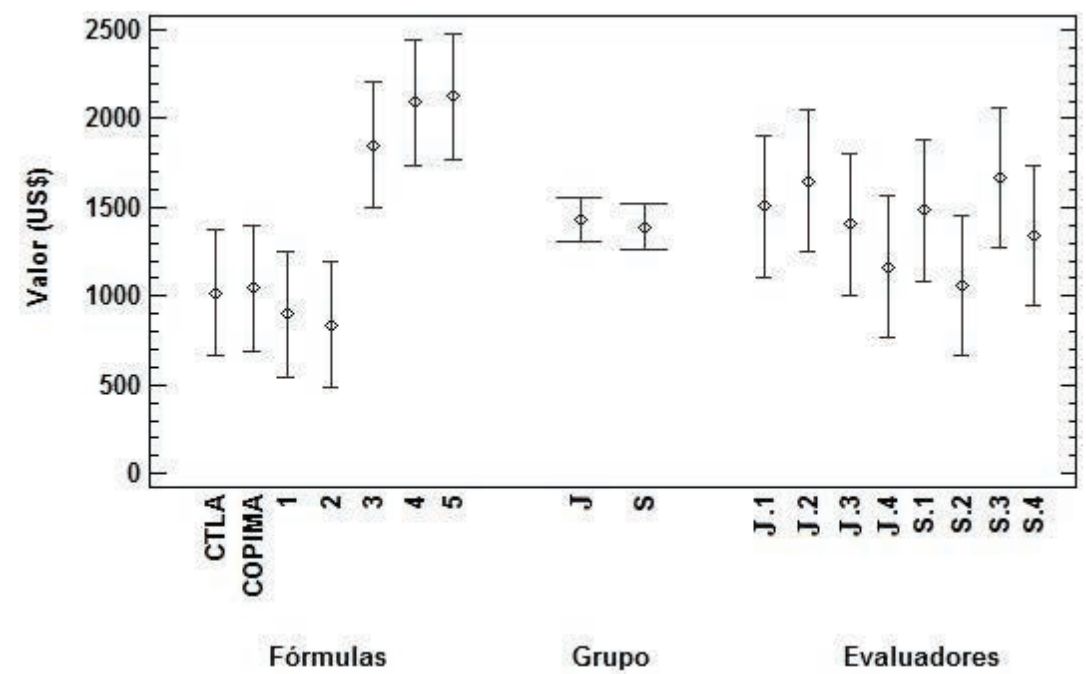

evaluadores

A) Valor (US\$)

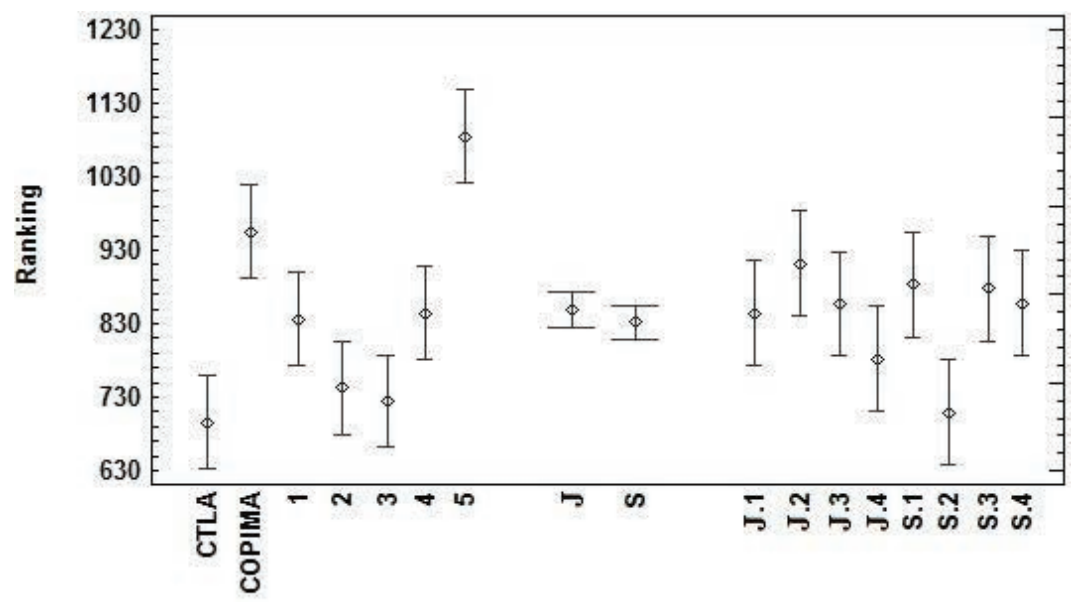

Fórmulas

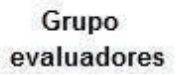

Evaluadores

B) Ranking

Figura 1. Media y error estándar del valor (US\$) y ranking por fuente de variación.

Media and standard error of value (US\$) and ranking by source of variation

Cuadro 5. Prueba de Tukey HSD del valor y ranking por grupo de evaluadores.

Tukey HSD test of value and ranking by group of evaluators.

\begin{tabular}{ccccc}
\hline $\begin{array}{c}\text { Grupo de } \\
\text { evaluadores }\end{array}$ & $\begin{array}{c}\text { Valor } \\
\text { promedio (US\$) }\end{array}$ & $\begin{array}{c}\text { Grupos } \\
\text { homogéneos del valor }\end{array}$ & $\begin{array}{c}\text { Ranking } \\
\text { promedio }\end{array}$ & $\begin{array}{c}\text { Grupos } \\
\text { homogéneos del ranking }\end{array}$ \\
\hline Júnior & $1.431,3$ & $\mathrm{G}$ & 848,9 & $\mathrm{H}$ \\
Sénior & $1.389,9$ & $\mathrm{G}$ & 832,1 & $\mathrm{H}$ \\
\hline
\end{tabular}


Diferencia entre evaluadores. En el análisis paramétrico del valor no se encontraron diferencias significativas entre los evaluadores (estadígrafo de 1,35, $P>0,05$ ), mientras que y sí las hubo cuando se analizó el ranking (estadígrafo de 3,81, $P<0,01$ ), conformándose dos grupos (grupo J y $\mathrm{K}$ del cuadro 6), aunque de los ocho tasadores sólo uno (Sénior.2) presentó diferencias significativas con el resto de los evaluadores, con menores valoraciones, ubicándose en el menor ranking promedio.

\section{DISCUSIÓN}

Los resultados monetarios muestran que las variables que componen las fórmulas siguen siendo la principal fuente de dispersión de sus valores (Watson 2002, Contato-Carol et al. 2008, Ponce-Donoso et al. 2012, 2013, Ponce-Donoso y Vallejos-Barra 2016), así como el modelo base que las sustenta (Randrup 2005).

La dispersión de los valores y posición en el ranking también muestran que las fórmulas presentan capacidad de discriminar el árbol, lo que las hace más efectivas al momento de valorar árboles, aspecto identificado por Watson (2002) y destacado por Contato-Carol et al. (2008), Grande-Ortiz et al. (2012) y Ponce-Donoso et al. (2012 y 2013). Sin embargo, Watson (2002) hace hincapié en el hecho que si las diferencias entre evaluadores son muy grandes, la credibilidad del método podría disminuir, sugiriendo que no debería ser más del doble. Al respecto, las fórmulas evaluadas no presentaron diferencias entre evaluadores como grupo, aunque el Sénior.2 presentó en la evaluación individual un valor promedio bajo, mostró una diferencia del $57 \%$ respecto del valor promedio más alto (Sénior.3).

El uso de las fórmulas CTLA y COPIMA resultaron ser buenos patrones de comparación para proponer nuevas fórmulas de aplicación, ya que permiten que los análisis de varianza aplicados logren discriminar cuál o cuáles de las cinco fórmulas propuestas se adaptan de mejor manera a los criterios de selectividad, lo que generó grupos de fórmula al aplicar la prueba de Tukey.
Los montos de las valoraciones (cuadro 2) muestran alta dispersión y algunas particularidades en ciertas fórmulas. Así, existe al menos un valor mínimo en las fórmulas 2, 3, 4, 5 y CTLA, que resulta insuficiente para reemplazar el árbol en el caso que exista pérdida total, mientras que los valores máximos de la fórmula 3, 4, 5 y CTLA resultan demasiado altos para hacer factible su aplicación en un Juzgado de Policía Local nacional, si se considera el ingreso medio de la población y el criterio de selectividad usado para proponer una fórmula. El coeficiente de variación evidencia la dispersión de la valoración con las distintas fórmulas, aquella con valores extremos (mínimo o máximos) presentan los mayores coeficientes (fórmulas 2, 3, 4 y CTLA), mientras que las fórmulas 1, 5 y COPIMA evidencian menores dispersiones. Asimismo, al transformar el valor a un Ranking se mantiene la tendencia en el coeficiente de variación.

En general las fórmulas propuestas se ajustan a la sugerencia de Cullen (2002) en términos que al analizar los valores y ranking de los evaluadores como grupo, no presentan diferencias; mientras que entre evaluadores, tampoco hubo diferencias para valores, pero se conformaron dos grupos en el ranking, siendo en este caso un evaluador sénior el único que presenta diferencias con el resto de los tasadores. Lo anterior es un hallazgo importante, que se diferencia con anteriores estudios (Watson 2002, Contato-Carol et al. 2008, Ponce-Donoso et al. 2012, 2013) ya que permite afirmar que las fórmulas propuestas pueden ser usadas por profesionales con poca (júnior) o mucha experiencia (sénior), lo que reduciría el peso de los denominados aspectos subjetivos de la fórmula (Caballer 1999, Watson 2002, Price, 2003, Tyrväinen et al. 2003, PonceDonoso et al. 2009), cuestión positiva si se vinculan estas labores a los requerimientos de los municipios, que muchas veces se ven impedidos de contar con profesionales experimentados en este campo, tal como lo indicaran Ponce y Piedrahita (2009). Por ello, el diseño aplicado permitió minimizar o eliminar las diferencias asociadas a las fórmulas entre evaluadores, ya sea analizado de manera

Cuadro 6. Prueba de Tukey HSD del valor y ranking por evaluadores.

Tukey HSD test of value and ranking by evaluators.

\begin{tabular}{ccccc}
\hline \multirow{2}{*}{ Evaluadores } & $\begin{array}{c}\text { Valor } \\
\text { promedio (US\$) }\end{array}$ & $\begin{array}{c}\text { Grupos } \\
\text { homogéneos del valor }\end{array}$ & $\begin{array}{c}\text { Ranking } \\
\text { promedio }\end{array}$ & $\begin{array}{c}\text { Grupos } \\
\text { homogéneos del ranking }\end{array}$ \\
\hline Júnior.1 & $1.505,0$ & $\mathrm{I}$ & 844,2 & $\mathrm{~J} \mathrm{~K}$ \\
Júnior.2 & $1.648,6$ & $\mathrm{I}$ & 912,4 & $\mathrm{~K}$ \\
Júnior.3 & $1.405,6$ & $\mathrm{I}$ & 857,1 & $\mathrm{~K}$ \\
Júnior.4 & $1.166,0$ & $\mathrm{I}$ & 781,8 & $\mathrm{~J} \mathrm{~K}$ \\
Sénior.1 & $1.484,5$ & $\mathrm{I}$ & 882,9 & $\mathrm{~K}$ \\
Sénior.2 & $1.062,0$ & $\mathrm{I}$ & 709,6 & $\mathrm{~J}$ \\
Sénior.3 & $1.668,4$ & $\mathrm{I}$ & 877,6 & $\mathrm{~K}$ \\
Sénior.4 & $1.343,4$ & $\mathrm{I}$ & 858,4 & $\mathrm{~K}$ \\
\hline
\end{tabular}


individual o en grupo, lo cual reduce la brecha asociada a la experiencia del tasador como fuente de variación.

El desempeño de las fórmula usadas como base de comparación, tal como muestran los estudios de Ponce-Donoso et al. (2009, 2012, 2013) y Ponce-Donoso y Vallejos-Barra (2016), COPIMA vuelve a tomar valores medios, aunque no logró agruparse con CTLA en el ranking, como señalan Ponce-Donoso y Vallejos-Barra (2016), aunque sí lo hace en términos de valor (cuadro 4).

$\mathrm{Al}$ analizar la mediana de los valores y teniendo en cuenta los criterios de elegibilidad señalados, las fórmulas 1 y 2 se agrupan con CTLA y COPIMA (grupo A) (cuadro 4, figura 1A). En el caso de las medianas analizadas en el ranking, CTLA y COPIMA se ubican en grupos diferentes; CTLA lo hace con las fórmulas 2 y 3 (grupo C) con valores medios bajos, mientras que COPIMA lo hace con las fórmulas 1 y 4 (grupo E), presentado valores medios altos. Las fórmulas 1, 2, 3 y 4 también conforman otro grupo (grupo D). Estos resultados permiten desechar la fórmula 5 propuesta, por no cumplir el criterio de agruparse en torno a alguna de las fórmulas usadas como base de comparación. Los resultados también señalan que efectivamente no existen diferencias entre evaluadores según su experiencia o profesión, aunque sí se identificaron dos grupos homogéneos de evaluadores individuales; sin embargo, no se pudo identificar algún tipo de patrón o causa en términos de su formación o experiencia con el uso de las formulas, lo que en todo caso no implica restricciones de su uso.

Al tener como premisa los criterios de selección, esto es una fórmula que incorpore variables ambientales, un valor monetario máximo y mínimo acorde a las condiciones locales, factibilidad de aplicación por municipios, utilizable por tasadores con y sin experiencia, con capacidad para discriminar las diferencias propias de los árboles y tener un mejor desempeño que las fórmulas de comparación y propuestas, el mejor método para valorar el arbolado urbano en Chile central corresponde a la fórmula 1, denominada: valoración monetaria del arbolado urbano (VaMA), por lo que se sugiere a los municipios de Chile central incluirla en sus ordenanzas, con la finalidad de contar con una herramienta eficaz y efectiva al momento de valorar monetariamente el arbolado urbano en áreas de uso público.

\section{CONCLUSIONES}

Se rechaza la hipótesis nula debido a la existencia de diferencias significativas entre las medianas para las fuentes de variación correspondiente a fórmulas, tanto en el valor como en el ranking; rechazándose también la hipótesis nula correspondiente al análisis del valor del tasador individual.

Se acepta la hipótesis nula debido a la no existencia de diferencias significativas entre las medianas para las fuentes de variación correspondiente a grupo de evaluadores, tanto en el valor como en el ranking; aceptándose también la hipótesis nula correspondiente al análisis del ranking del tasador individual.
De las cinco fórmulas propuestas evaluadas, la fórmula 1 presenta comparativamente el mejor desempeño, considerando tanto los resultados del análisis estadístico, los estadígrafos usados y el cumplimiento de las condiciones de borde establecidas. Asimismo, junto con incorporar variables ambientales, presenta un mejor desempeño que las fórmulas CTLA y COPIMA.

Frente a la inexistencia de fórmulas para valorar monetariamente el arbolado urbano en Chile central que incorporen variables ambientales, se propone la fórmula 1 para ser usada en actuaciones de valoración monetaria de árboles urbanos en Chile central, proveyendo a gobiernos locales, profesionales e investigadores una herramienta efectiva y eficaz, para determinar el valor monetario del arbolado urbano tanto público como privado.

\section{AGRADECIMIENTOS}

Los autores agradecen al Fondo Nacional de Ciencia y Tecnología de Chile (FONDECYT) por el apoyo recibido para la ejecución del Proyecto 1130264.

\section{REFERENCIAS}

AEPJP (Asociación Española de Parques y Jardines Públicos, ES). 2007. Norma Granada. Método para valoración de árboles y arbustos ornamentales. $3^{\mathrm{a}}$ ed. Madrid, España. AEPJP. 53 p.

BCN (Biblioteca del Congreso Nacional, CL). Reportes Estadísticos Distritales y Comunales 2013. Consultado 10 ene. 2014. Disponible en: http://reportescomunales.bcn.cl.

Caballer V. 1999. Valoración de Árboles. Madrid, España. Mundi-Prensa. 247 p.

Chueca J. 2001. La Norma Granada: un método de valoración económica de los árboles ornamentales. Consultado oct. 2007. Disponible en: www.drac.com/pers/chueca/Granada. htm.

Contato-Carol ML, E Ayuga-Tellez, MA Grande-Ortiz. 2008. A comparative analysis of methods for the valuation of urban trees in Santiago del Estero, Argentina. Spanish Journal of Agricultural Research 6(3): 314-352.

CTLA (Council of Tree \& Landscape Appraiser, US). 2000. Guide for Plant Appraisal. 9th ed. Champaign, IL, USA. International Society of Arboriculture. 143 p.

Cullen S. 2002. Tree appraisal: can depreciation factors be rated greater than 100\%? Journal of Arboriculture 28(3): 153158.

Cullen S. 2005. Tree appraisal: chronology of North American industry guidance. Journal of Arboriculture 31(4): 157162.

Dobbs C, FJ Escobedo, WC Zipperer. 2011. A framework for developing urban forest ecosystem services and goods indicators. Landscape and Urban Planning 99(3-4): 196-206.

Escobedo FJ, T Kroeger, J Wagner. 2011. Urban forest and pollution mitigation: analyzing ecosystem services and disservices. Environmental Pollution 159(8-9): 2078-2087.

Flook R. 1996. A Standard Tree Evaluation Method - STEM. Journal of the New Zealand Institute of Horticulture 1(3).

Grande-Ortiz M, M Ayuga-Tellez, M Contato-Carol. 2012. 
Methods of tree appraisal: a review of their features and application possibilities. Arboriculture \& Urban Forestry 38(4): 130-140.

Haase D, N Larondelle, E Andersson, M Artmann, S Borgström, J Breuste, E Gomez-Baggethum, A Gren, Z Hamstead, R Hansen, N Kabish, P Kremer, J Langemeyer, E Lorance, T McPhearson, S Pauleit, S Qureshi, N Schwarz, A Voigt, D Wurster, T Elmqvist. 2014. A quantitative review of urban ecosystem service assessments: concepts, models, and implementation. Ambio 43(4): 413-433.

Hegedüs A, M Gaál, R Bérces. 2011. Tree appraisal methods and their application first results in one Budapest's districts. Applied Ecology and Environmental Research 9(4): 411-423.

Konijnendijk C, S Syaka, T Randrup, L Schipperijn. 2004. Urban and peri-urban forestry in the development context: strategic and implementation. Journal of Arboriculture 30(5): 269-276.

Konijnendijk C, N Kjell, T Randrup, J Schipperijn. 2005. Urban Forest and Trees. Amsterdam, Holanda. Springer Verlag. $520 \mathrm{p}$.

Moore GM, T Arthur. 1992. Amenity tree evaluation: A revised method. In Arthur $\mathrm{T}$ ed. The Scientific Management of Plants in the Urban Environment. Proceedings of the Burnley Centenary Conference. Centre for Urban Horticulture. Melbourne, Australia. p: 166-171.

Pauleit S, N Jones, G García-Marín, J García-Valdecantos, L Rivière, L Vidal-Beaudet, M Bodson, T. Randrup. 2002. Tree establishment practice in towns and cities: results from European survey. Urban Forest and Urban Greening 1(2): 83-96.

Ponce-Donoso M, P Piedrahita. 2009. Valoración económica del arbolado urbano en 28 comunas de Chile. Quebracho 17(1, 2): 89-100.

Ponce-Donoso M, L Moya, O Bustos-Letelier. 2009. Evaluation of formula for the appraisal of urban trees in municipalities of Chile. Scientia Forestalis 37(83): 321-329.

Ponce-Donoso M, O Vallejos-Barra, G Daniluk-Mosquera. 2012. Comparación de fórmulas chilenas e internacionales para valorar el arbolado urbano. Bosque 33(1): 69-81.

Ponce-Donoso M, O Vallejos-Barra, C Avilés-Palacios. 2013. Comparison of seven Chilean formulae for urban tree appraisal. Agrociencia 47(7): 723-737.

Ponce-Donoso M, O Vallejos-Barra. 2016. Valoración de árboles urbanos, comparación de fórmulas. Revista de la Facultad de Ciencias Agrarias de la Universidad Nacional de Cuyo 48(2): 195-208.

Price C. 2003. Quantifying the aesthetic benefits of urban forestry. Urban Forest and Urban Greening 1(3): 123-134.

Randrup TB. 2005. Development of Danish model for plant appraisal. Journal of Arboriculture 31(3): 114-123.

Roy S, J Byrne, C Pickering. 2012. A systematic quantitative review of urban tree benefits, costs, and assessment methods across cities in different climatic zones. Urban Forestry and Urban Greening 11(4): 351-363.

Tyrväinen L. 2001. Economic valuation of urban forest benefits in Finland. Journal of Environmental Management 62(1): 75-92.

Tyrväinen L, H Silvennoinen, O Kolehmainen. 2003. Ecological and aesthetic value in urban forest management. Urban Forest and Urban Greening 1(3): 135-149.

Watson G. 2002. Comparing formula methods of tree appraisal. Journal of Arboriculture 28(1): 11-18.

Recibido: 05.09.16

Aceptado: 16.11 .16 